\title{
Labor induction in term nulliparous women with premature rupture of membranes: oxytocin versus dinoprostone
}

\author{
Nur Gozde Kulhan, Mehmet Kulhan
}

Department of Gynecology and Obstetrics, Erzincan University, Erzincan, Turkey

Submitted: 5 March 2018

Accepted: 8 May 2018

Arch Med Sci 2019; 15 (4): 896-901

DOI: https://doi.org/10.5114/aoms.2018.76115

Copyright @ 2018 Termedia \& Banach

\section{Abstract}

Introduction: Premature rupture of the membranes (PROM) refers to rupture of the fetal membranes prior to the onset of regular uterine contractions. Premature rupture of the membranes continues to be one of the most vexing issues of obstetrics due to increased maternal and fetal morbidity and mortality. Many studies have focused on how management should be in these cases. The purpose of this study was to investigate whether dinoprostone ( $\mathrm{PGE}_{2}$ analogue) administration is necessary for cervical ripening and labor induction in term women with premature rupture of membranes (PROM) and to compare maternal and neonatal outcomes between oxytocin usage and dinoprostone usage in PROM.

Material and methods: A total of 224 nulliparous singleton pregnant women at term, with $P R O M \geq 12 \mathrm{~h}$, vertex presentations, no prior uterine surgery, reactive non-stress test and Bishop scores $\leq 6$ (unfavorable cervixes) were reviewed. Participants were divided into two groups as oxytocin and dinoprostone groups. The primary outcome was vaginal delivery within $24 \mathrm{~h}$.

Results: The women in the oxytocin group were significantly younger than in the dinoprostone group ( $22.85 \pm 4.10$ years vs. $25.99 \pm 4.94$ years; $p=0.001)$. There were significant differences in vaginal delivery rates within 24 h. It was 72 of $112(64.3 \%)$ vs. 53 of $112(47.3 \%), p=0.023$ for oxytocin and dinoprostone groups, respectively.

Conclusions: Vaginal dinoprostone appears to be a relatively inefficient method of inducing labor compared with oxytocin in term pregnancies with PROM and unfavorable cervixes. However, dinoprostone may maintain uterine contractions as effectively as oxytocin once uterine contractions are established.

Key words: dinoprostone, induction of labor, oxytocin, prostaglandin $E_{2}$, premature rupture of the membranes.

\section{Introduction}

Premature rupture of the membranes (PROM) refers to rupture of the fetal membranes prior to the onset of regular uterine contractions [1]. Premature rupture of the membranes continues to be one of the most vexing issues of obstetrics due to increased maternal and fetal morbidity and mortality. Many studies have focused on how management should be in these cases. The major question regarding the management of patients with PROM is whether to allow them to enter labor spontaneously or to induce labor. A 2017 systematic review of 23 randomized trials

\author{
Corresponding author: \\ Nur Gozde Kulhan PhD \\ Department of Gynecology \\ and Obstetrics \\ Erzincan University \\ 24030 Erzincan, Turkey \\ Phone: +90 5054918090 \\ E-mail: gozdemirel1@ \\ hotmail.com
}


of women with PROM at $\geq 37$ weeks of gestation that compared pregnancy outcome of planned early intervention versus expectant management found that patients benefitted from induction of labor compared with expectant management [2]. For women who are not contraindicated for labor and vaginal delivery, there is no consensus on the method of labor induction. Meta-analyses of randomized trials have not demonstrated a clear benefit from initial use of any prostaglandin other than oxytocin in women with PROM, including those with unfavorable cervixes [3, 4]; however, data for the latter subgroup are limited. Recent guidance support the use of oxytocin as the firstline labor induction method in PROM [5]. Prostaglandin $E_{2}\left(P G E_{2}\right)$ vaginal inserts have been shown to be safe and efficacious in promoting cervical ripening in women with post-term pregnancies and low Bishop scores. However, there are insufficient data related to the efficacy and safety of $\mathrm{PGE}_{2}$ in term pregnancies complicated with PROM. Only a few small trials have evaluated pre-induction cervical ripening in women with PROM and an unfavorable cervix [6, 7]. Nulliparous and unfavorable cervixes needing labor induction remain a clinical challenge.

The objective of this study was to investigate whether dinoprostone ( $\mathrm{PGE}_{2}$ analogue) administration is necessary for cervical ripening and labor induction in women with PROM and to compare maternal and neonatal outcomes between those women who were inducted with oxytocin and dinoprostone.

\section{Material and methods}

The study started after approval of the ethics committee of our hospital. A total of 224 nulliparous singleton pregnant women at term, with $P R O M \geq 12 \mathrm{~h}$, vertex presentations, and Bishop scores $\leq 6$ (unfavorable cervixes) were reviewed. All subjects gave written informed consent. Patients with regular uterine contraction, ultrasonographically calculated fetal weight over $4500 \mathrm{~g}$, placenta/vasa previa or unexplained vaginal bleeding, past uterine surgeon, prolabial umbilical cord, signs of chorioamnionitis, and contraindications for prostaglandin or oxytocin administration (i.e. heart disease, glaucoma, bronchial asthma, severe renal insufficiency) were exclusion criteria.

Subjects were divided into two groups: group 1 , labor induction with oxytocin $(n=112)$; group 2 , labor induction with the dinoprostone vaginal slow release system $(n=112)$. The cases in both protocol groups were compared in terms of the following parameters: age, maternal weight, maternal body mass index (BMI), anemia and other antenatal risk factors, initial Bishop score, time between onset of treatment and active phase, time between onset of treatment and delivery, change in Bishop score in the first $12 \mathrm{~h}$, mean blood loss in labor, intrapartum fever, fetal tachycardia, uterine hypertonicity, uterine tachysystole, uterine hyperstimulation, birth weight, meconium-stained amnion in labor, fetal sex, first minute Apgar score, fifth minute Apgar score, newborn intensive care need, indication for cesarean delivery.

In group 1, oxytocin (Synpitan forte $5 \mathrm{U}$, Deva, Turkey) was prepared as $10 \mathrm{U}$ of oxytocin $/ 500 \mathrm{ml}$ $5 \%$ dextrose. Induction was started at $4 \mathrm{mU} / \mathrm{min}$ and increased by $4 \mathrm{mU} / \mathrm{min}$ every $15 \mathrm{~min}$ to a maximum of $32 \mathrm{mU} / \mathrm{min}$ [8]. In group 2 a $10 \mathrm{mg}$ dinoprostone containing vaginal pessary slow-release system (Propess, Vitalis) was placed digitally in the posterior fornix of the vagina according to the manufacturer's guidelines. The Propess insert was left in situ for $12 \mathrm{~h}$; active labor was determined when cervical dilatation was $4 \mathrm{~cm}$. The Bishop score was assessed and documented every hour by the same clinician. Not achieving active labor despite $12 \mathrm{~h}$ of continuous treatment with the Propess insert was considered as induction failure. To avoid bias and confusion during the interpretation of data, no other labor induction methods were used, and for these cases, "failed induction" was reported as the indication for cesarean delivery. The usual prophylactic regimen was an initial dose of $2 \mathrm{~g}$ of ampicillin intravenously followed by $1 \mathrm{~g}$ of ampicillin intravenously every $4 \mathrm{~h}$ until delivery.

The primary outcome was percentage of vaginal deliveries within $24 \mathrm{~h}$. Secondary outcomes were induction-to-delivery interval, mode of delivery, time to complete dilatation, uterine hyperstimulation, change in Bishop score at $12 \mathrm{~h}$, meconium-stained amniotic fluid, estimated intrapartum blood loss, maternal fever, and neonatal outcomes such as neonatal admission for special care and Apgar score.

\section{Statistical analysis}

SPSS 20 (IBM Corp. Released 2011. IBM SPSS Statistics for Windows, Version 20.0. Armonk, NY: IBM Corp.) was used to analyze the data. $P<0.05$ and $p<0.01$ were taken as significance levels. For discrete and continuous variables, descriptive statistics (mean, standard deviation, number and percentile) were used. In addition, the homogeneity of the variances, which is one of the prerequisites of parametric tests, was checked by Levene's test. The assumption of normality was tested via the Shapiro-Wilk test. To compare the differences between the groups, Student's $t$ test was used when the parametric test prerequisites were fulfilled, and the Mann-Whitney $U$ test was used when such prerequisites were not fulfilled. Categorical data were analyzed using Fisher's exact test and 
the $\chi^{2}$ test. In cases in which the expected counts for inclusion were not met in less than $20 \%$ of the cells, the Monte Carlo simulation method was used and the values were determined.

\section{Results}

Demographic characteristics such as age, gestational week, BMI, parity and antenatal risk factors are presented in Table I. The women in the oxytocin group were younger than the women in the dinoprostone group. $(22.85 \pm 4.10$ vs. 25.99 \pm 4.94 years; $p=0.001$ ). There were no significant differences between the two groups in gestational week, maternal weight and antenatal risk factors except anemia, while there was a significant difference in maternal age and BMI. Pre-induction Bishop scores were similar in both groups. There were significant differences in the predefined primary outcomes including vaginal delivery rates within $24 \mathrm{~h}$; they were 72 of $112(64.3 \%)$ vs. 53 of $112(47.3 \%), p=0.023$ for oxytocin and dinoprostone respectively. There were also significant difference in secondary outcomes. Labor induction to delivery interval was longer in the dinoprostone group, but this difference was not statistically significant $(p=0.519)$. The time from rupture of membranes to initiation of oxytocin and dinoprostone was not significantly different between the groups ( $12.5 \pm 1.15 \mathrm{~h}$ vs. $12.83 \pm 2.30 \mathrm{~h}, p=0.078)$. There was no significant difference in the incidence of fetal heart rate abnormalities, tachysystole, or intrapartum fever between two groups, but estimated blood loss was significantly higher in the dinoprostone group than the oxytocin group $(533.39 \pm 183.71 \mathrm{ml}$ vs. $454.64 \pm 189.63 \mathrm{ml}$, $p=0.002)$. Cesarean delivery rates were significantly higher in the dinoprostone group than the oxytocin group $(52.7 \%$ vs. $35.7 \%, p=0.015)$. Labor contractility was established slightly earlier in the oxytocin group than in the dinoprostone group of patients. For those treated with dinoprostone, mean uterine activity reached a peak within 6-8 $\mathrm{h}$ after the medication. In the oxytocin group, the mean uterine activity rose steadily and reached a peak at 2-3 h after treatment. Continuous infusion of oxytocin was required until delivery. One hour after the start of treatment the mean frequency of contractions per 10 min was slightly lower in the dinoprostone treated group of patients (oxytocin 3.2, PGE2 2.2 contractions per $10 \mathrm{~min}$ ). After approximately $4 \mathrm{~h}$ a steady state had been reached, with a mean frequency of contractions per $10 \mathrm{~min}$ in the oxytocin group of 4.5 and in the dinoprostone group of 5.2. Also the intensity of contractions increased more rapidly in the group of patients induced by oxytocin. Atypical contractility patterns were significantly more common in dinoprostone induced labor than in labor induced by oxytocin. Uterine hyperstimulation was observed more often in patients treated with dinoprostone $(22.3 \%$ vs. $7.14 \%, p=0.001)$. Uterine hyperactivity was similar between two groups. Clinical and neonatal outcomes of both groups are summarized in Table II.

\section{Discussion}

Premature rupture of the membranes continues to be one of the most vexing issues of obstetrics due to increased maternal and fetal morbidity and mortality. Many studies have been conducted on how management should be in these cases, and immediate delivery of the fetus, especially over the $36^{\text {th }}$ gestational week, is the recommended approach. It can be expected for a while in other cases, but this waiting period is also limited to $12 \mathrm{~h}$ in many centers [1]. In the case of prolonged

Table I. Demographic characteristics of study population

\begin{tabular}{|c|c|c|c|}
\hline Parameter & Oxytocin $(n=112)$ & Dinoprostone $(n=112)$ & $P$-value \\
\hline Age [years] & $22.85 \pm 4.10$ & $25.99 \pm 4.94$ & $0.001^{* *}$ \\
\hline Maternal weight [kg] & $69.5 \pm 11.15$ & $70.15 \pm 6.12$ & 0.588 \\
\hline Maternal BMI [kg/m²] & $28.57 \pm 2.83$ & $27.04 \pm 1.96$ & $0.001^{* *}$ \\
\hline Gestational age [week] & $38.85 \pm 1.19$ & $39.10 \pm 1.33$ & 0.140 \\
\hline Parity & $1(0)$ & $1(0)$ & - \\
\hline \multicolumn{4}{|l|}{ Antenatal risk factors: } \\
\hline None & $84(47.2 \%)$ & $94(52.8 \%)$ & \\
\hline Gestational diabetes & 0 & 0 & $0.003^{* *}$ \\
\hline Anemia & $28(68.3 \%)$ & $13(31.7 \%)$ & \\
\hline Hypertension & 0 & $5(100 \%)$ & \\
\hline
\end{tabular}


Table II. Clinical and neonatal outcomes of the study population

\begin{tabular}{|c|c|c|c|}
\hline Parameter & Oxytocin $(n=112)$ & Dinoprostone $(n=112)$ & $P$-value \\
\hline Pre-induction Bishop score & $1.64 \pm 1.45$ & $1.76 \pm 1.40$ & 0.098 \\
\hline Bishop score change in $12 \mathrm{~h}$ & $5.21 \pm 2.1$ & $5.66 \pm 3.53$ & 0.244 \\
\hline PROM to induction interval [h] & $12.5 \pm 1.15$ & $12.83 \pm 2.30$ & 0.078 \\
\hline Induction to active labor onset [h] & $5.69 \pm 5.39$ & $9.45 \pm 4.25$ & $0.001^{* *}$ \\
\hline Induction to delivery [h] & $12.5 \pm 7.15$ & $13.03 \pm 5.07$ & 0.519 \\
\hline Vaginal delivery & $72(64.3 \%)$ & $53(47.3 \%)$ & $0.023^{*}$ \\
\hline Cesarean delivery & $40(35.7 \%)$ & $59(52.7 \%)$ & $0.015^{*}$ \\
\hline \multicolumn{4}{|l|}{ Indication for cesarean delivery: } \\
\hline Fetal distress & $12(10.7 \%)$ & $17(15.2 \%)$ & \\
\hline Cephalopelvic disproportion & $20(17.9 \%)$ & $10(8.9 \%)$ & $0.001^{\star *}$ \\
\hline Failed induction & $8(7.1 \%)$ & $32(28.6 \%)$ & \\
\hline Mean Apgar score at $1 \mathrm{~min}$ & $7.92 \pm 0.45$ & $7.87 \pm 0.42$ & 0.367 \\
\hline Mean Apgar score at $5 \mathrm{~min}$ & $8.92 \pm 0.45$ & $8.91 \pm 0.38$ & 0.875 \\
\hline Mean birth weight $[g]$ & $3140 \pm 383.50$ & $3003.97 \pm 487.15$ & $0.021^{*}$ \\
\hline \multicolumn{4}{|l|}{ Fetal sex: } \\
\hline Male & $68(60.7 \%)$ & $61(54.5 \%)$ & 0.417 \\
\hline Female & $44(39.3 \%)$ & $51(45.5 \%)$ & \\
\hline Meconium-stained liquor in labor & $1.10 \pm 0.31$ & $1.22 \pm 0.41$ & $0.019^{*}$ \\
\hline Neonatal admission for special care & $16(14.3 \%)$ & $8(7.14 \%)$ & 0.129 \\
\hline Maternal fever & $32(28.6 \%)$ & $42(37.5 \%)$ & 0.155 \\
\hline Delivery blood loss [ml] & $454.64 \pm 189.63$ & $533.39 \pm 183.71$ & $0.002^{*}$ \\
\hline Uterine hypertonicity & $8(7.14 \%)$ & $9(8 \%)$ & 0.801 \\
\hline Uterine tachysystole & $12(10.7 \%)$ & $17(15.2 \%)$ & 0.320 \\
\hline Uterine hyperstimulation & $8(7.14 \%)$ & $25(22.3 \%)$ & $0.001^{\star *}$ \\
\hline
\end{tabular}

PROM, the obstetric balance has been proven to be impaired in the negative direction [9]. It is suggested that labor should be induced when there are no spontaneous uterine contractions. The purpose of induction is to reduce the risk of operative delivery and fetal complications. The most preferred approach for induction is oxytocin infusion, but several studies have reported that using oxytocin without cervical ripening increases cesarean rates in such cases with an unfavorable cervix [10]. Unfortunately, the cases of PROM are often accompanied by a low cervical score ( $\leq 4$ according to Bishop score) [11]. It has been proposed that PGE and $P G E_{2}$ can be administered vaginally to stimulate cervical ripening in term pregnancies with low Bishop scores or complicated with PROM $[1,3]$.

Prostaglandin $E_{2}$ is an efficacious agent that shortens the time from induction to delivery, im- proves success rates and reduces morbidity associated with labor induction [12]. Prostaglandin $\mathrm{E}_{2}$ was first used by Calder and Embray in 1973 for induction of labor [13]. Initially, the controlled release dinoprostone ovule (Propess) was reported to be contraindicated in patients with PROM, but it has been recommended for use in patients with PROM since 2006. The number of studies that have investigated the efficacy and safety of dinoprostone ovules in PROM is limited. In our study, it was found that the use of dinoprostone ovule (Propess) vaginally was effective in providing cervical ripening as well as in maintaining uterine contractions required for vaginal birth and without the need for oxytocin. There are some reports showing that this agent significantly shortens the latent phase of delivery. In the present study, labor induction to active labor interval and time from 
induction to delivery were found to be longer in the dinoprostone compared to the oxytocin group ( $p=0.001, p=0.519$, respectively). In one study, in term women with PROM oxytocin use shortened the induction to active labor onset compared to dinoprostone. On the other hand, another study comparing dinoprostone with expectant management in the treatment of PROM at term found that the time of dilatation was shorter in the dinoprostone group $[14,15]$.

We also found that dinoprostone affects the rate of cesarean delivery or indications. In this context, the rates of cesarean delivery for the dinoprostone and oxytocin groups were $52.7 \%$ and $35.7 \%$, respectively. In a study conducted by Larrañaga-Azcárate et al., the rate of cesarean section was found to be significantly lower in patients treated with controlled-release dinoprostone ovules compared to the spontaneously monitored group $(9.3 \%$ vs. $17.6 \%, p=0.04$ ) [14]. However, we observed higher cesarean rates in patients treated with dinoprostone than those treated with oxytocin ( $p=$ $0.015)$. This may be related to a greater number of active phase labor abnormalities in the dinoprostone group. In the dinoprostone group, cesarean deliveries were performed for failed induction, fetal distress and cephalopelvic disproportion with the rate of $28.6 \%, 15.2 \%, 8.9 \%$ respectively. One of the factors that might affect the cesarean birth rate is fetal birth weight; however, it was found to be $3140 \pm 383.50 \mathrm{~g}$ in the oxytocin group and 3003.97 $\pm 487.15 \mathrm{~g}$ in the dinoprostone group $(p=0.021)$.

In a study by Ramsey et al., it was found that the fetal cardiotocographic abnormalities associated with PGE2 were less frequent and less severe than those associated with PGE1 [16]. However, the present study demonstrated that the changes in cardiotocographic measurements were similar in the PGE 2 and oxytocin groups, while uterine hyperstimulation was observed more often in patients treated with dinoprostone $(22.3 \%$ vs. $7.14 \%, p=0.001$ )

We used meconium-stained amniotic fluid and $1^{\text {st }}$ and $5^{\text {th }}$ min Apgar score data to evaluate fetal asphyxia. While there was no difference in $1^{\text {st }}$ and $5^{\text {th }}$ min Apgar scores between groups, meconium-stained amniotic fluid was observed more frequently in the dinoprostone group compared to the oxytocin group $(22.3 \%$ vs. $10.7 \%$, $p=0.019)$. Abdelazim et al. compared patients who received antenatal oxytocin and those who underwent elective cesarean section without oxytocin; they reported that the fetal outcomes of patients who underwent spontaneous labor or those who received oxytocin before cesarean section were better than those of patients who did not receive oxytocin before cesarean section [17].

The major limitation of this study is its retrospective design. Patients were allocated to a par- ticular treatment (dinoprostone or oxytocin) by their attending obstetrician, rather than being randomly assigned. Because an individual clinician's management style affects their choice of induction agent as well as the decision to proceed to cesarean delivery, this may have introduced selection bias and influenced our results. However, at our institution most obstetricians practice in large groups that share labor and delivery coverage. This more closely resembles a hospitalist model and reduces the impact of any one obstetrician's management style on our results.

In conclusion, vaginal dinoprostone appears to be a relatively inefficient method of inducing labor compared with oxytocin in term pregnancies with PROM and unfavorable cervixes. However, PGE may maintain uterine contractions as effectively as oxytocin once uterine contractions are established.

\section{Conflict of interest}

The authors declare no conflict of interest.

\section{References}

1. ACOG Committee on Practice Bulletins-Obstetrics. Premature rupture of membranes. Practice Bulletin No. 172. American College of Obstetricians and Gynecologists. Obstet Gynecol 2016; 128: e165-77.

2. Middleton P, Shepherd E, Flenady V, et al. Planned early birth versus expectant management (waiting) for prelabour rupture of membranes at term (37 weeks or more). Cochrane Database Syst Rev 2017; 1: CD005302.

3. Tan BP, Hannah ME. Prostaglandins versus oxytocin for prelabour rupture of membranes at term. Cochrane Database Syst Rev 2000; 2: CD000159.

4. Lin MG, Nuthalapaty FS, Carver AR, et al. Misoprostol for labor induction in women with term premature rupture of membranes: a meta-analysis. Obstet Gynecol 2005; 106: 593-601.

5. ACOG Committee on Practice Bulletins-Obstetrics. ACOG Practice Bulletin No. 80: premature rupture of membranes. Clinical management guidelines for obstetriciangynecologists. Obstet Gynecol 2007; 109: 1007-19.

6. Lange AP, Secher NJ, Nielsen FH, Pedersen GT. Stimulation of labor in cases of premature rupture of the membranes at or near term. A consecutive randomized study of prostaglandin E2-tablets and intravenous oxytocin. Acta Obstet Gynecol Scand 1981; 60: 207-10.

7. McQueen D, Neilson JP, Whittle MJ. Pre-labour rupture of membranes with an unripe cervix: a random trial of management. J Obstet Gynaecol 1990; 10: 495-8.

8. ACOG technical bulletin. Induction of labor. Number 217-Dec 1995 American college of Obstetricians Gynecologists. Int J Gynaecol Obstet 1996; 53: 65-72.

9. Everest NJ, Jacobs SE, Davis PG, Begg L, Rogerson S. Outcomes following prolonged preterm premature rupture of the membranes. Arch Dis Child Fetal Neonatal Ed 2008; 93: F207-11.

10. Thomas J, Fairclough A, Kavanagh J, Kelly AJ. Vaginal prostaglandin (PGE2 and PGF2a) for induction of labour at term. Cochrane Database Syst Rev 2014; 6: CD003101. 
11. Boulvain M, Kelly A, Lohsec stan C, Lirion D. Mechanical methods for induction of labor. Cochrane Database Syst Rev 2003; 189: 1031-5.

12. Parashi S, Bonabi NB, Rashidi A. Re: oxytocin induction of labour: a comparison of two protocols. Aust N Z J Obstet Gynaecol 2005; 45: 540.

13. Fletcher HM, Mitchell S, Simeon D, Frederich S, Brown D. Intravaginal misoprostol as a cervical ripening agent. Br J Obstet Gynecol 1993; 100: 641-4.

14. Kunt C, Kanat-Pektas M, Gungor AN, et al. Randomized trial of vaginal prostaglandin E2 versus oxytocin for labor induction in term premature rupture of membranes. Taiwan J Obstet Gynecol 2010; 49: 57-61.

15. Larrañaga-Azcárate C, Campo-Molina G, Perez-Rodrigez $A F$, Ezcurdia-Gurpegui M. Dinoprostone vaginal slow-release system (Propess ${ }^{\circledR}$ ) compared to expectant manage-ment in the active treatment of premature rupture of the membranes at term: impact on maternal and fetal out-comes. Acta Obstet Gynecol Scand 2008; 87: 195-200.

16. Ramsey PS, Meyer L, Walkes BA, et al. Cardiotocographic abnormalities associated with dinoprostone and misoprostol cervical ripening. Am J Obstet Gynecol 2005; 105: 85-90.

17. Abdelazim I, Farghali MMM, Elbiaa AAM, et al. Impact of antenatal oxytocin infusion on neonatal respiratory morbidity associated with elective cesarean section. Arch Med Sci 2017; 13: 629-34. 